\title{
Biochemical markers of bone turnover in patients with spinal metastases after resistance training under radiotherapy - a randomized trial
}

Harald Rief ${ }^{{ }^{*}}$, Georg Omlor ${ }^{3}$, Michael Akbar ${ }^{3}$, Thomas Bruckner², Stefan Rieken', Robert Förster ${ }^{1}$, Ingmar Schlampp ${ }^{1}$, Thomas Welzel ${ }^{1}$, Tilman Bostel ${ }^{1}$, Heinz Jürgen Roth ${ }^{4}$ and Jürgen Debus ${ }^{1}$

\begin{abstract}
Background: To compare the effects of resistance training versus passive physical therapy on bone turnover markers (BTM) in the metastatic bone during radiation therapy (RT) in patients with spinal bone metastases. Secondly, to evaluate an association of BTM to local response, skeletal-related events (SRE), and number of metastases.

Methods: In this randomized trial, 60 patients were allocated from September 2011 to March 2013 into one of the two arms: resistance training (Arm A) or passive physical therapy (Arm B) with thirty patients in each arm during RT. Biochemical markers such as pyridinoline (PYD), desoxy-pyridinoline (DPD), bone alkaline phosphatase (BAP), total amino-terminal propeptide of type I collagen (PINP), beta-isomer of carboxy-terminal telopeptide of type I collagen (CTX-I), and cross-linked N-telopeptide of type I collagen (NTX) were analyzed at baseline, and three months after RT.

Results: Mean change values of PYD and CTX-I were significantly lower at 3 months after RT ( $p=0.035$ and $p=0.043$ ) in Arm A. Importantly, all markers decreased in both arms, except of PYD and CTX-I in arm B, although significance was not reached for some biomarkers. In arm A, the local response was significantly higher $(p=0.003)$ and PINP could be identified as a predictor for survivors (OR 0.968, 95\%Cl 0.938-0.999, $p=0.043$ ). BAP (OR 0.974, 95\%Cl 0.950-0.998, $p=0.034)$ and PINP (OR 1.025, 95\% Cl 1.001-1.049, $p=0.044$ ) were related with an avoidance of SRE.

Conclusions: In this group of patients with spinal bone metastases, we were able to show that patients with guided resistance training of the paravertebral muscles can influence BTM. PYD and CTX-I decreased significantly in arm A. PINP can be considered as a complementary tool for prediction of local response, and PINP as well as BAP for avoidance of SRE.
\end{abstract}

Trial registration: Clinical trial identifier NCT 01409720. August 2, 2011.

Keywords: Bone metastases, Spine, Physical exercise, Biochemical markers, Resistance training

\section{Background}

Spinal bone metastases represent the most frequent site of skeletal metastasis [1], and radiotherapy (RT) is the most common treatment option of bone metastases in an advanced tumour disease [2]. As a result of the alteration in bone remodeling activity in patients

\footnotetext{
* Correspondence: harald.rief@med.uni-heidelberg.de

'Department of Radiation Oncology, University Hospital of Heidelberg, Im

Neuenheimer Feld 400, 69120 Heidelberg, Germany

Full list of author information is available at the end of the article
}

with bone metastases, various markers of bone formation or resorption have been investigated as a measurement of activity of skeletal metastases. Balance between bone resorption and bone formation is required for maintenance of bone metabolism, and can be reflected by bone turnover markers (BTM). The usefulness of BTM as a tool for the diagnosis of bone metastases in many types of cancers has been investigated previously [3]. Previous studies have demonstrated that some BTM can indicate the existence of 
bone metastases [4], and have analyzed their relationship with clinical outcomes [5-9].

The two most clinically relevant markers of bone turnover in patients with skeletal metastases are bonespecific alkaline phosphatase (BAP) and N-terminal telopeptide of collagen type I (NTX). Total amino-terminal propeptide of type I collagen (PINP) and BAP are markers of bone formation; BAP has a linear relationship with osteoblast and osteoblastic precursor activity. NTX is a breakdown product of type I collagen produced by various proteolytic enzymes during bone resorption and dissolution of the organic bone matrix. Additionally, pyridinium cross-links pyridinoline (PYD) and desoxy-pyridinoline (DPD) are well-characterized markers for bone resorption. C-terminal cross-linking telopeptide of type I collagen (CTX-I) can be considered a complementary tool for prediction of clinical outcome as a marker of resorption. At the present time, although biochemical markers of bone turnover have shown some utility in clinical trials by predicting clinical outcomes such as death or development of SREs, they are not routinely used in clinical practice. Questions remain as to how clinicians should best use these markers to select and time appropriate treatments among patients with bone metastases. The use of these markers may, in the future, better allow physicians to selectively treat patients with bone metastases.

In our recent work, we could show the feasibility of resistance training in patients with spinal bone metastases under RT [10]. Importantly, we demonstrated that the bone density in metastases of the resistance training arm was significantly higher than in the control arm after 3 months. Therefore, we hypothesized that resistance training concomitant to RT could influence bone metabolism, and would be reflected by BTM.

The aim of this randomized trial was to compare the effect of resistance training concomitant to RT versus RT only on BTM, such as BAP, PINP, CTX-I, PYD, DPD, and NTX in patients with spinal bone metastases. Secondly, we evaluated an association of these markers as predicted factors for local response, prevention of SRE, and number of metastases.

\section{Methods}

\section{Settings and patients}

This is a randomized, controlled, two-armed intervention trial. A block randomization approach with block size 6 was used to ensure that the two intervention groups were balanced. After the baseline measurements, the patients with stable bone metastases were assigned to the respective treatment groups on a 1:1 basis according to the randomization list. Arm A (intervention group, resistance training) and arm B (control group, passive physical therapy) each consisted of 30 patients.
The blood and urine parameters were measured before start of RT $\left(t_{0}\right)$ (day of the first fraction), and after three months $\left(t_{2}\right)$ on an empty stomach. The primary endpoint was to compare BTM after 3 months in patients treated with resistance training concomitant to RT versus RT only in spinal bone metastases. Secondary endpoint was to evaluate predictive bone markers for local response, prevention of SRE, and number of metastases. Local response of metastasis was assessed on the basis of computed tomography. Positive local response was defined as complete or partial re-calcification on the basis of computed tomography observation at 3 months after RT. The definition of SRE was the first of any of the following events: pathological fractures, severe pain (increase of more than 2 points according to numeric rating scale), hypercalcaemia, and spinal cord compression. Number of metastases was classified in 1 or $>1$ (solitary vs. multiple). The data of the patient records were collected by the authors. Patient characteristics are shown in Table 1.

Inclusion criteria were an age of 18 to 80 years, a Karnofsky performance score $[11] \geq 70$, written consent to participate, and already initiated bisphosphonate therapy. Patients were excluded if they presented with concomitant pathologies that could interfere in the evaluation of bone turnover markers, such as bone metabolic disorders, Paget's disease of bone, hyperparathyroidism, thyroid abnormalities, abnormal intestinal absorption, or hepatic or renal dysfunction. In all cases, anticancer treatment could be changed as clinically indicated throughout the course of the study. The patients were subjected to a staging of their vertebral column within the context of the computed tomography $(\mathrm{CT})$ designed to plan the radiation schedule prior to enrolment into the trial. In this examination metastases were classified as "stable" or "unstable". This was diagnosed independently by a specialist for radiology as well as by a specialist for orthopedic surgery. The specifications for an unstable vertebral body were tumor occupancy more than $60 \%$ of the vertebral body, and pedicle destruction [12]. Only a metastasis classified by both specialists as "stable" was suggested eligible for inclusion. Patients with significant neurological or psychiatric disorders were excluded. The study was approved by the Heidelberg Ethics Committee (Nr. S-316/2011).

\section{Radiotherapy}

RT was performed in the Radiooncology Department of the Heidelberg University Clinic. After virtual simulation was performed to plan the radiation schedule, RT was carried out over a dorsal photon field of the 6MV energy range. Primary target volume (PTV) covered the specific vertebral body affected as well as the ones immediately above and below. In Arm A, 24 patients (80\%) were treated with $10 \times 3 \mathrm{~Gy}$, three patients $(10 \%)$ with $14 \times$ 
Table 1 Patient characteristics at baseline

\begin{tabular}{|c|c|c|c|c|c|}
\hline & & Intervention grou & & Control group ( $n$ & \\
\hline & & $n$ & $\%$ & $\bar{n}$ & $\%$ \\
\hline Age (years) & & & & & \\
\hline & Mean (SD) & $61.3+/-10.1$ & & $64.1+/-10.9$ & \\
\hline Gender & & & & & \\
\hline & Male & 14 & 46.7 & 19 & 63.3 \\
\hline & Female & 16 & 53.3 & 11 & 36.7 \\
\hline Karnofsky-index (median, range) & & $80(70-100)$ & & $80(70-100)$ & \\
\hline Primary site & & & & & \\
\hline & Lung cancer & 12 & 9.2 & 8 & 26.6 \\
\hline & Breast cancer & 5 & 16.7 & 6 & 20.1 \\
\hline & Prostate cancer & 5 & 16.7 & 9 & 30.1 \\
\hline & Melanoma & 1 & 3.3 & 1 & 3.3 \\
\hline & Renal cancer & 1 & 3.3 & 2 & 6.7 \\
\hline & Other & 6 & 20.1 & 4 & 13.4 \\
\hline Localization metastases & & & & & \\
\hline & Thoracic & 17 & 56.7 & 14 & 46.7 \\
\hline & Lumbar & 9 & 30.0 & 13 & 43.3 \\
\hline & Thoracic and lumbar & 2 & 6.7 & 2 & 6.7 \\
\hline & Sacrum & 2 & 6.7 & 1 & 3.3 \\
\hline Number metastases & & & & & \\
\hline & Mean (range) & $1.4(2-4)$ & & $1.7(1-5)$ & \\
\hline & Solitary & 22 & 73.3 & 18 & 60.0 \\
\hline & Multiple & 8 & 26.7 & 12 & 40.0 \\
\hline Type of metastases & & & & & \\
\hline & osteoblast & 9 & 30.0 & 10 & 33.3 \\
\hline & osteolytic & 21 & 70.0 & 20 & 66.7 \\
\hline Size of metastasis & & & & & \\
\hline & Mean (SD) & $318.6+/-230.0$ & & $380.7+/-193.6$ & \\
\hline Distant metastases at baseline & & & & & \\
\hline & Visceral & 12 & 40.0 & 5 & 16.7 \\
\hline & brain & 3 & 10.0 & 3 & 10.0 \\
\hline & lung & 7 & 23.3 & 4 & 13.3 \\
\hline & tissue & 8 & 26.7 & 6 & 20.0 \\
\hline Hormonotherapy & & 10 & 33.3 & 16 & 53.3 \\
\hline Immunotherapy & & 7 & 23.3 & 5 & 16.7 \\
\hline Chemotherapy & & 25 & 83.3 & 20 & 66.7 \\
\hline Previous SRE & & 9 & 30.0 & 13 & 43.3 \\
\hline
\end{tabular}

Abbreviation: SD standard deviation

2.5 Gy, and three patients $(10 \%)$ with $20 \times 2$ Gy. In Arm B the dose fractions for 28 patients $(93.4 \%)$ were $10 \times$ $3 \mathrm{~Gy}$, for one patient (3.3\%) $14 \times 2.5 \mathrm{~Gy}$, and for one patient $(3.3 \%) 20 \times 2$ Gy. The median individual dose in all patients was 3 Gy (range 2-3 Gy), the median total dose 30 Gy (range 20-35 Gy). The individual and total doses were decided separately for each individual patient, depending on the histology, the patient's general state of health, and on the current staging and the corresponding prognosis.

\section{Study interventions}

The interventions commenced on the same day as RT and were performed on each day of RT treatment 
(Monday through Friday) over a 2-week period, independent of the number of fractions. During the 2-week RT period, the patients in the resistance training arm (Arm A) performed the exercises under the guidance of a physiotherapist. The patients were then instructed to practice the training in their homes three times a week and continued the resistance training themselves until the last investigation after three months, and conducted the documentation in form of a training diary. The resistance training lasted approx. 30 min, the passive physical therapy (Arm B) approx. $15 \mathrm{~min}$. Since the site of the bone metastases differed from patient to patient, three different exercises were enacted to ensure an even isometric resistance training of the paravertebral muscles. Patients in the control arm (Arm B) received passive physical therapy in form of breathing exercises also for a period of 2 weeks. This was conducted so that these patients were not discouraged in comparison to the intervention arm, and for avoidance of a high dropout rate. A detailed report of the intervention and its application has already been published [13].

\section{Statistical analysis}

Bone marker values were presented in terms of mean and standard deviation (SD). The possible difference between arms at 3 months was calculated using ANCOVA with arm as factor and baseline value as covariate. Therefore as all surviving patients completed all surveys we assumed that 23 patients in Arm A and 24 patients in Arm B were eligible for the analysis. Multivariate binary logistic regression models were fitted to quantify the degree of association between potential predictors of markers at baseline according to local response (yes/no), prevention of SRE (yes/no), and number of metastases (1/more than one). Group had been included as a covariate in the analysis. Arm A with 23 survived patients was the reference. Level of significance was set to 0.05 for all tests. All statistical analyses were done using SAS software Version 9.4 (SAS Institute, Cary, NC, USA).

\section{Analytic methods}

Samples were centrifuged at $3.500 \mathrm{rpm}$ for $10 \mathrm{~min}$ after drawing, serum obtained and stored at $-80^{\circ}$. First morning void of urine was collected, mixed and an aliquot of $10 \mathrm{ml}$ stored at $-80{ }^{\circ} \mathrm{C}$. Samples shipped on dry ice to the laboratory (Labor Limbach, Heidelberg, Germany) and again stored at $-80{ }^{\circ} \mathrm{C}$ until analysis.

Total aminoterminal propeptide of type I collagen (PINP; bone formation; interassay coefficient of variation [CV]: $2.7 \%$ ), C-terminal cross-linking telopeptide of type I collagen (CTX-I; bone resorption; CV: $3.1 \%$ ) and intact parathyroid hormone (iPTH; CV: $1.4 \%$ ) were measured by means of an automated electrochemiluminescence immunoassay (ECLIA; Modular Analytics E170, Roche
Diagnostics, Penzberg, Germany). Bone-specific alkaline phosphatase (BAP; bone formation; CV: 5,2 \%) was measured by means of a spectrophotometric immunoassay (IDS-ISYS Ostase BAP; Immunodiagnostic Systems Ltd [IDS Ltd], Boldon, Tyne \& Wear, UK) on the fully automated immunoassay system (IDS-ISYS (Immunodiagnostic Systems Ltd [IDS Ltd], Boldon, Tyne \& Wear, UK). Pyridinoline (PYD) and deoxypyridinoline (DPD; bone resorption; $\mathrm{CV}: 12.5 \%$ ) were assayed by high performance liquid chromatography (HPLC) $[14,15]$. Analysis required first a sample extraction, hydrolysis and automated column-chromatographic pre-purification. To avoid any effect of different dilutions of urine, Pyr and Dpyr data are expressed against gram of creatinine. Cross-linked Ntelopeptides of type I collagen (NTx; bone resorption; CV: $12.8 \%$ ) was determined with an commercially available ELISA (Osteomark Ntx; Wampole Laboratories, Princeton NJ, USA).

All laboratory analyses were done in two batches after completion of the sample collection. To further reduce imprecision of measurement, all samples were analyzed utilizing one reagent lot. Tests and instruments were run strictly in accordance with the guidelines given by the manufacturer and were subject to continuous maintenance and service according to the laboratories standard operating procedures for good laboratory practice. Samples were thawed at the day of analysis at ambient temperature, mixed on a head-over-head mixer and centrifuged before measurement.

\section{Results}

From September 2011 to March 2013, consecutive 80 patients with a histologically confirmed cancer of any primary and spinal bone metastases of the thoracic or lumbar segments, or of the sacral region were considered in the Radiooncology Department of the Heidelberg University Clinic. Fifteen patients were excluded due to unstable metastases, and five patients declined to participate in the study. Sixty patients fulfilled the inclusion and were enrolled into the trial (Fig. 1). Arms were balanced at baseline without group differences, particularly number of metastases and tumor size (Table 1). Seven patients $(23.3 \%)$ died in arm A within the first 12 weeks following RT, six patients in arm B (20.0\%) died within 3 months. The mean follow-up was 3.3 months for both groups. All surviving patients completed all surveys. Mortality did not differ between groups.

Mean change values of PYD and CTX-I were significantly lower at 3 months after RT $(p=0.035$ and $p=$ 0.043 ) in arm A. DPD showed a tendency with a lower mean change value, but not significant. No changes were seen between the arms in BAP, NTX, and PINP. Importantly, all markers decreased in both arms, except of PYD 
and CTX-I in arm B, although significance was not reached for some biomarkers (Table 2).

In arm $\mathrm{A}$, the local response was significantly higher [73.9\% $(n=17)$ vs. $25 \%(n=6)](p=0.003)$. At first, PINP could be identified as a predictor of local response $(p=$ $0.043)$. BAP $(p=0.034)$ and PINP $(p=0.044)$ could be identified as a predictor for avoidance of SRE. No marker could be detected for solitary metastasis (Table 3).

\section{Discussion}

Bone metastases are a very frequent secondary diagnosis associated with an advanced tumor disease, with the vertebral column being the most frequent localization [2, 10]. The usefulness of BTM as tool for the diagnosis of bone metastases in many types of cancers has been investigated previously [3]. Many studies have analyzed their relationship with clinical outcomes $[7,16]$ as well as their clinical importance $[17,18]$. However, there is still no concensus among these studies as to which BTM is the ideal marker.

In the current work, all BTM in arm A decreased after 3 months, especially the mean change values of PYD and CTX-I were significantly lower at 3 months after RT due to resistance training. BTM in arm B showed also decreased values after 3 months except of PYD and CTX-I, but not so distinctive as in arm A. Importantly, local response as re-calcification in metastasis was improved in arm A due to resistance training. In our recent work, we could show that bone density was significantly increased in metastasis 3 months after start of resistance training concomitant to RT [19]. This factor may influence the BTM and was demonstrated in our results. Therefore, PINP could be detected as a strong marker for bone formation after combined treatment with resistance training concomitant to RT.

Determination of CTX-I is recommended for monitoring the efficacy of antiresorptive therapy. Patients with renal cell carcinoma who died or progressed had higher baseline B-CTX levels and those who experienced SRE during follow-up showed high BAP levels [20]. However, in our results we could not found any useful data in normal/abnormal values of BTM. A multicenter study found that increased values of BAP and NTX were associated with increased risk of SRE, and disease progression in patients with breast cancer, prostate cancer, and other solid tumors, treated with bisphosphonates [21]. These markers of bone turnover have been used clinically to predict the risk of SREs and disease outcomes. According our results, we could identify BAP and PINP for avoidance of SRE. In addition, biochemical markers of bone turnover may prove useful in the monitoring of patients on bisphosphonate treatment for bone metastases. One review [22] of 121 patients with skeletal metastases from variety of solid tumors revealed that mean values of NTX were consistently elevated in patients experiencing SREs compared with those who did not. An additional study [23] showed in 441 patients that patients with high NTX or BAP levels had a greater incidence of SREs compared with patients with low levels of NTX or BAP. Patients with increased NTX or BAP at

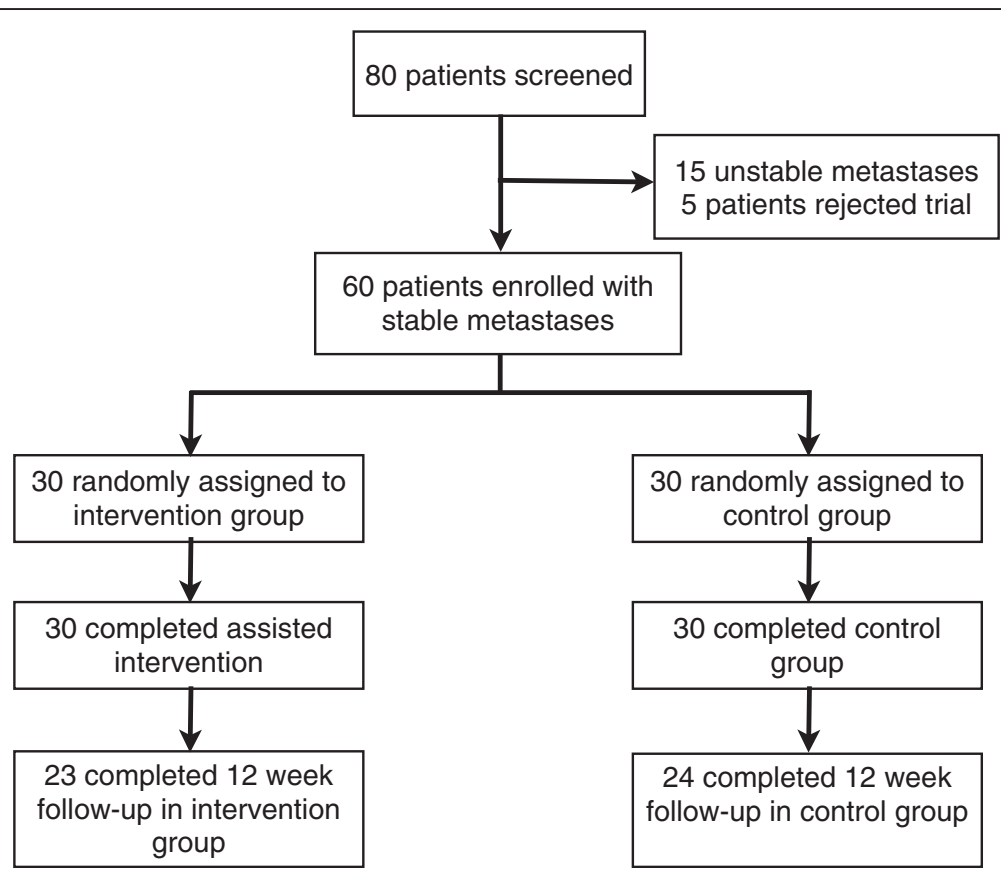

Fig. 1 Flow of participants through the trial 
Table 2 Results of bone turnover markers

\begin{tabular}{|c|c|c|c|c|c|c|c|c|c|c|c|}
\hline \multirow[b]{3}{*}{ Parameter } & \multirow[b]{3}{*}{ Reference value } & \multicolumn{4}{|l|}{$\operatorname{Arm} A$} & \multicolumn{4}{|l|}{ Arm B } & \multirow{3}{*}{$\begin{array}{l}\text { Difference between arms } \\
\text { LS means } \\
\text { Difference }(95 \% \mathrm{Cl})\end{array}$} & \multirow[b]{3}{*}{$p$-value } \\
\hline & & \multicolumn{2}{|c|}{ baseline, $n=23$} & \multicolumn{2}{|c|}{3 months, $n=23$} & \multicolumn{2}{|c|}{ baseline, $n=24$} & \multicolumn{2}{|c|}{3 months, $n=24$} & & \\
\hline & & mean & SD & mean & SD & mean & SD & mean & SD & & \\
\hline PYD & $160-280 \mu \mathrm{g} / \mathrm{g} \mathrm{Cr}$ & 375.63 & 206.20 & 299.89 & 173.88 & 402.00 & 199.01 & 405.50 & 187.32 & $-90.92(-175.19 ;-6.65)$ & 0.035 \\
\hline DPD & $26-65 \mu \mathrm{g} / \mathrm{g} \mathrm{Cr}$ & 67.30 & 39.64 & 51.20 & 35.81 & 86.10 & 78.85 & 72.88 & 37.11 & $-18.20(-38.84 ; 2.45)$ & 0.082 \\
\hline CTX-I & $<0.5484 \mathrm{ng} / \mathrm{ml}$ & 0.29 & 0.27 & 0.20 & 0.23 & 0.39 & 0.37 & 0.42 & 0.45 & $-0.10(-0.20 ;-0.003))$ & 0.043 \\
\hline BAP & $6-15 \mu \mathrm{g} / \mathrm{l}$ & 56.89 & 92.31 & 31.46 & 63.31 & 44.17 & 47.32 & 36.52 & 37.58 & $-12.10(-31.51 ; 7.31)$ & 0.215 \\
\hline NTX & $5.4-24.2 \mathrm{nmol} / \mathrm{l}$ & 19.09 & 7.21 & 17.42 & 7.98 & 21.42 & 9.36 & 20.38 & 9.81 & $-0.87(-3.84 ; 2.10)$ & 0.556 \\
\hline PINP & $15-59 \mu \mathrm{g} / \mathrm{l}$ & 67.54 & 40.69 & 49.50 & 41.43 & 87.96 & 51.98 & 66.77 & 39.18 & $-6.94(-26.81 ; 12.93)$ & 0.485 \\
\hline
\end{tabular}

*Results of ANCOVA

This table shows the results of bone turnover markers of both groups at baseline and 3 months after RT. Differences are presented within and between groups Abbreviation: $L S$ means least square means

baseline had an increased risk for SRE, shorter time to first event, disease progression and death. As NTX levels have been shown to correlate with response to bisphosphonate treatment. In our data, we could not show any correlation of NTX to local response, SRE, and number of metastases, but we were able to confirm the relation

Table 3 Results of multivariate binary logistic regression analysis of bone markers

\begin{tabular}{|c|c|c|c|}
\hline Local response (no local response) & OR & $95 \% \mathrm{Cl}(\mathrm{OR})$ & $p$-value \\
\hline Arm (A - reference) & 0.033 & $0.004-0.311$ & 0.003 \\
\hline PYD & 1.000 & $0.992-1.008$ & 0.986 \\
\hline DPD & 1.023 & $0.974-1.074$ & 0.371 \\
\hline CTX-I & 10.550 & $0.003->100$ & 0.569 \\
\hline BAP & 1.011 & $0.996-1.025$ & 0.142 \\
\hline NTX & 0.870 & $0.688-1.101$ & 0.245 \\
\hline PINP & 0.968 & $0.938-0.999$ & 0.043 \\
\hline Prevention of SRE $(S R E=0)$ & OR & 95 \% Cl (OR) & $p$-value \\
\hline Arm (A - reference) & 3.302 & $0.877-12.431$ & 0.077 \\
\hline PYD & 0.998 & $0.994-1.002$ & 0.373 \\
\hline DPD & 1.006 & $0.982-1.031$ & 0.633 \\
\hline CTX-I & 0.323 & $0.001-81.336$ & 0.689 \\
\hline BAP & 0.974 & $0.950-0.998$ & 0.034 \\
\hline NTX & 0.988 & $0.861-1.134$ & 0.865 \\
\hline PINP & 1.025 & $1.001-1.049$ & 0.044 \\
\hline Solitary metastasis $(n=1)$ & OR & 95 \% Cl (OR) & $p$-value \\
\hline Arm (A - reference) & 1.692 & $0.477-6.007$ & 0.416 \\
\hline PYD & 1.001 & $0.997-1.005$ & 0.512 \\
\hline DPD & 1.004 & $0.980-1.029$ & 0.761 \\
\hline CTX-1 & 0.359 & $0.001->100$ & 0.723 \\
\hline BAP & 0.997 & $0.985-1.009$ & 0.592 \\
\hline NTX & 1.083 & $0.939-1.249$ & 0.275 \\
\hline PINP & 0.998 & $0.981-1.016$ & 0.829 \\
\hline
\end{tabular}

Abbreviation: SRE skeletal-related events to BAP with prevention of SRE. Zoledronic acid normalizes or maintains normal NTX levels in most patients with bone metastases. All of our participants were treated with bisphosphonates. Our data presented no differences between arms of NTX.

However, a PINP could be identified as a predictor for local response and avoidance of SRE. In this novel study, our results showed that resistance training concomitant to RT can even enhance an effect on BTM.

Since all patients were in advanced stages of their cancer, $40 \%$ of the patients in either group were lost to follow-up due to progressive disease and subsequently death. Further limitations of the study were the relatively small sample size, the variety of primary tumors and patient conditions, and the exclusion of patients presenting with cervical spine metastases.

Among the strengths of our novel and original study were the randomized design and the very first presentation of bone turnover markers in combined treatment of resistance training concomitant to $\mathrm{RT}$ in patients with spinal bone metastases.

\section{Conclusion}

In this group of patients with spinal bone metastases, we were able to show that patients with guided resistance training of the paravertebral muscles can influence BTM. PYD and CTX-I decreased significantly in arm A. PINP can be considered as a complementary tool for prediction of local response, and PINP as well as BAP for avoidance of SRE. More studies in larger groups of patients are necessary for further confirmatory research.

\section{Ethics approval}

The study was approved by the Heidelberg Ethics Committee (Nr. S-316/2011).

\section{Consent for publication}

Not applicable. 


\section{Availability of data and materials}

The dataset supporting the conclusions of this article is included within the article

\section{Abbreviations}

ANCOVA: analysis of covariance; BAP: bone alkaline phosphatase; BTM: bone turnover markers; CT: computed tomography; CTX-l: beta-isomer of carboxyterminal telopeptide of type I collagen; CV: coefficient of variation; DPD: desoxy-pyridinoline; ECLIA: automated electrochemiluminescence immunoassay; Gy: Gray; HPLC: high performance liquid chromatography; iPTH: intact parathyroid hormone; NTX: cross-linked N-telopeptide of type I collagen; PINP: total amino-terminal propeptide of type I collagen; PTV: Primary target volume; PYD: pyridinoline; RT: radiation therapy; SD: standard deviation; SRE: skeletal related events.

\section{Competing interests}

The authors declare that they have no competing interests.

\section{Authors' contributions}

$H R$ and JD developed and planned this trial. TB is responsible for statistical considerations/basis of the analysis. GO, MA, and TW estimated the stability of bone metastases. HR, SR, RF and TBo performed the examinations and RT supervisions. HJR estimated the evaluation of BTM. HR made the data collection. HR performed the physical exercise. All authors read and approved the final manuscript.

\section{Acknowledgements}

The authors thank all of the study participants for their great effort.

\section{Funding}

No funding was used for this study.

\section{Author details}

${ }^{1}$ Department of Radiation Oncology, University Hospital of Heidelberg, Im Neuenheimer Feld 400, 69120 Heidelberg, Germany. ${ }^{2}$ Department of Medical Biometry, University Hospital of Heidelberg, Im Neuenheimer Feld 305, 69120 Heidelberg, Germany. ${ }^{3}$ Department of Orthopaedics and Trauma Surgery, University Hospital of Heidelberg, Schlierbacherstrasse 120a, 69118 Heidelberg, Germany. ${ }^{4}$ Department of Endocrinology/Oncology, Limbach Laboratory Heidelberg, Im Breitspiel 15, 69126 Heidelberg, Germany.

Received: 9 December 2014 Accepted: 13 March 2016

Published online: 17 March 2016

\section{References}

1. Coleman RE. Metastatic bone disease: Clinical features, pathophysiology and treatment strategies. Cancer Treat Rev. 2001;27:165-76.

2. Lutz S, Berk L, Chang E, et al. Palliative radiotherapy for bone metastases: an ASTRO evidence-based guideline. Int J Radiol Oncol Biol Phys. 2011;79(4): 965-76.

3. Coleman R, Costa L, Saad F, et al. Consensus on the utility of bone markers in the malignant bone disease setting. Crit Rev Oncol Hematol. 2011;80(3): 411-32.

4. Zhao H, Han KL, Wang ZY, et al. Value of C-telopeptide-cross-linked type I collagen, osteocalcin, bone specific alkaline phosphatase and procollagen type I N-terminal propeptide in the diagnosis and prognosis of bone metastasis in patients with malignant tumors. Med Sci Monit. 2011;17(11): 626-33.

5. Jung $K$, Lein $M$, Ringsdorf $M$, et al. Diagnostic and prognostic validity of serum bone turnover markers in metastatic renal cell carcinoma. J Urol. 2006;176:1326-31.

6. Kamiya N, Suzuki H, Yano M, et al. Implications of serum bone turnover markers in prostate cancer patients with bone metastasis. Urology. 2010; 75(6):1446-51.

7. Mountzios G, Terpos E, Syrigos K, et al. Markers of bone remodeling and skeletal morbidity in patients with solid tumors metastatic to the skeleton receiving the biphosphonate zoledronic acid. Transl Res. 2010;155(5):247-55.

8. Saad F, Eastham JA, Smith MR. Biochemical markers of bone turnover and clinical outcomes in men with prostate cancer. Urol Oncol. 2012;30(4):369-78.
9. Zaghloul MS, Boutrus $\mathrm{R}$, El-Hossieny $\mathrm{H}$, et al. A prospective, randomized, placebo-controlled trial of zoledronic acid in bony metastatic bladder cancer. Int J Clin Oncol. 2010;15(4):382-9.

10. Rief $\mathrm{H}, \mathrm{Omlor} \mathrm{G}$, Akbar $\mathrm{M}$, et al. Feasibility of isometric spinal muscle training in patients with bone metastases under radiation therapy - first results of a randomized pilot trial. BMC Cancer. 2014;14:67.

11. Karnofsky DA, Burchenal JH (1949) The Clinical Evaluation of Chemotherapeutic Agents in Cancer. In: MacLeod CM (Ed), Evaluation of Chemotherapeutic Agents. New York, pp191-205.

12. Taneichi H, Kaneda K, Takeda N, et al. Risk factors and probability of vertebral body collapse in metastases of the thoracic and lumbar spine. Spine. 1997;22:239-45.

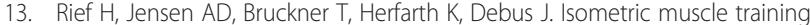
of the spine musculature in patients with spinal bony metastases under radiation therapy. BMC Cancer. 2011;11:482

14. Vesper HW, Audain C, Woolfitt A, et al. High-performance liquid chromatography method to analyze free and total urinary pyridinoline and desoxypyridinoline. Anal Biochem. 2003;318:204-11.

15. Seibel MJ, Lang M, Geilenkeuser WJ. Interlaboratory variation of biochemical markers of bone turnover. Clin Chem. 2001:47:1443-50.

16. Jung $K$, Miller $K$, Wirth $M$, et al. Bone turnover markers as predictors of mortality risk in prostate cancer patients with bone metastases following treatment with zoledronic acid. Eur Urol. 2011;59:604-12

17. Coleman RE, Lipton A, Roodman GD, et al. Metastasis and bone loss: advancing treatment and prevention. Cancer Treat Rev. 2010;36:615-20.

18. Armstrong AJ, Eisenberger MA, Halabi $\mathrm{S}$, et al. Biomarkers in the management and treatment of men with metastatic castration-resistant prostate cancer. Eur Urol. 2012;61:549-59.

19. Rief H, Petersen LC, Omlor $G$, et al. The effect of resistance training during radiotherapy on spinal bone metastases in cancer patients - a randomized trial. Radiother Oncol. 2014;112(1):133-9.

20. Alcaraz A, Gonzalez-Lopez R, Morote J, et al. Biochemical markers of bone turnover and clinical outcome in patients with renal cell and bladder carcinoma with bone metastases following treatment with zoledronic acid: The TUGAMO study. British J cancer. 2013;109:121-30.

21. Coleman RE, Major P, Lipton A, et al. Predictive value of bone resorption and formation markers in cancer patients with bone metastases receiving the bisphosphonate zoledronic acid. J Clin Oncol. 2005;23(22):4925-35.

22. Brown JE, Thomson CS, Ellis SP, et al. Bone resorption redicts for skeletal complications in metastatic bone disease. Br J Cancer. 2003;89:2031-7.

23. Brown JE, Cook RJ, Major P, et al. Bone turnover markers as predictors of skeletal complications in prostate cancer, lung cancer, and other solid tumors. JNCl. 2005;97:59-69.

\section{Submit your next manuscript to BioMed Central and we will help you at every step:}

- We accept pre-submission inquiries

- Our selector tool helps you to find the most relevant journal

- We provide round the clock customer support

- Convenient online submission

- Thorough peer review

- Inclusion in PubMed and all major indexing services

- Maximum visibility for your research

Submit your manuscript at www.biomedcentral.com/submit
C Biomed Central 